\title{
THE SELECTION OF BENCHMARKING PARTNERS FOR VALUE MANAGEMENT: AN ANALYTIC APPROACH
}

\author{
Qiping SHEN ${ }^{1}$ and Guiwen LIU $^{2}$ \\ 1 Department of Building and Real Estate, The Hong Kong Polytechnic University, Hung Hom, Kowloon, Hong \\ Kong. Tel: +852 2766 5817; Fax:+8522764 5131;E-mail: bsqpshen@inet.polyu.edu.hk \\ 2 Faculty of Construction Management and Real Estate, Chongqing University, Chongqing, China. Tel: $+(86) 23$ \\ 65120976; Email: gwliu@cqu.edu.cn
}

\begin{abstract}
Learning from the best in class organisations provides the necessary guarantee for success in benchmarking. Jlowever, the selection of ideal benchmarking partners is a complcx, time-consuming and multi-faceted decision-making process. This paper presents a selection model for the selection of Value Management (VM) benchmarking partners based on the principle of analytical hierarchy process (AHP). It is a key step in a research project aiming to develop a successful framcwork for implementing value management (VM) in China's construction industry. The application of Expert Choice software makes the building of comparison matrix, the calculation of consistency ratio and the determination of weighting more effective and accurate. The output of a benchmarking study suggests a selection model that is reliable and principles which can be used in other benchmarking activities.
\end{abstract}

\section{Keywords}

Benchmarking, Analytical Hierarchy Process, Value Management, Expert Choice

\section{INTRODUCTION}

Benchmarking is the search for best practices that will lead to breakthrough improvenents of an organisation's performance. (Adersen \& Pettersen, 1996). It is a concept that is an integral part of a comprehensive total-quality-management (TQM) effort. The history of benchmarking is fairly well documented by many writers (Camp, 1989; McNair and Rimmer, 1992; Spendolini, 1992; Watson, 1993). Xerox is given credit for inventing the concept through their practice of how it managed to close the performance gap with its Japanese competitors such as Canon in the late 1970s. There are slight differences in benchmarking definitions in existing literature depending on the focus and scope of application. Based on the benchmarking experience at Xerox, Camp (1989) provided the definition: "Benchmarking is the search for industry best practices that lead to superior performance". A more refined definition, which was developed by the International Benchmarking Clearing House Design Committee (APQC, 1993) and represented a consensus among 100 companies, is, "Benchmarking is a systematic and continuous measurement process; a process of continuously measuring and comparing an organisation's business against business leaders anywhere in the world to gain information which will help the organisation to take action to improve its performance".

The essentials of benchmarking are to learn from leading organisations and implement improvements (APQC, 1993). Identifying ideal benchmarking partners is a critical factor for the success of benchmarking. Obviously, if the performance of benchmarking partners do not represent the best in class, it is hard to learn something from them effectively and efriciently and also impossible for an organisation to make expected improvcment. However, the number of possible benchmarking candidates is so large that often make the identification and selection 
phase is a time- and effort-consuming procedure. The selection of ideal benchmarking partners always considers various attributes ranging from technical issues to cultural aspects. In order to improve the chances of successful partner selection for benchmarking, a number of authors argue that multi-attribute decision-making tools should be used (Razmi, et al, 1999; Zairi, 1994).

A research project was undertaken at the Hong Kong Polytechnic University to benchmark the best in class value management (VM) users for the development of a success ful implementation framework in China's construction industry. VM is a systematic, organized, function-oriented and multi-disciplinary team approach. Either the process or output of VM cannot be measured with a single attribute. Therefore, the selection of VM benchmarking partner is also a multiattribute, decision-making process. The paper presents an approach for the selection of VM benchmarking partners via a quantitative model - Analytic Hierarchy Process (AHP). This method shortened the duration to identify ideal benchmarking partners and ensured the quality of benchmarking output. It is also hoped that the method suggested in this paper can be applied by other researchers when they benchmark subjects involving both 'hard' and 'soft' aspects.

\section{BENCHMARKING VALUE MANAGEMENT IN CONSTRUCTION}

Benchmarking is a very structured process that consists of several steps to be taken. The process of benchmarking focusses on the issue of how learning can be made and systematically incorporated into the organization. Watson (1993) highlights that the process of benchmarking involves asking four key questions:

1) What should we benchmark?

2) Whom should we benchmark?

3) How do we performance the process?

4) How do they performance the process?

These steps are often provided as a model by a number of influential benchmarking authors (Camp, 1989; Watson, 1993; Andersen and Pettersen, 1996). One of the most widely cited process models of benchmarking was designed by Xerox (Camp, 1989). This consists of four steps i.e. planning, analysis, integration and action.

Following the argument of Garnett and Pickrell (2000), ultimate output or completed productoriented benchmarking will not produce meaningful results for the project-based activity. Therefore, the VM process, not the output of VM exercise, is initially selected as the subject for the benchmarking of VM. As for a traditional process benchmarking, it is important to measure the process through the collection of measurable process performance data. The performance gap between the organisation and the benchmarking partner indicates the scope of improvement for the organisation, which does not perform that process to the level identified.

In accordance with the research objectives of the project, however, the benchmarking in this research does not focus on indicating the performance gap of an organisation's VM practice from 'best practices' level, but concentrates on how to elicit and consolidate elements from thesc best in class VM practitioners for developing a VM implementation framework. As revealed by Male er al's (1998) work, it is not realistic to expect meaningful result derived from the benchmarking of VM only based on "hard" metrics since VM is a managerial rather than a technical approach. It is inevitable to encounter a large number of 'soft' issues in the benchmarking process. Unlike the traditional way of benchmarking between two partners, 
many partners are involved in this project and the research team is in effect a third-party coordinator, not one of the benchmarking partners in the benchmarking process. In this respect, the benchmarking approach may be regard as a third-party benchmarking which has been described by Fisher et al. (1995).

According to objectives of the research project and previous recommendation of other researchers, the approach for benchmarking VM is developed on Xerox's model and tailored according to the characteristics of VM. It contains four steps as follows:

\section{Planning stage}

The objective of the benchmarking VM is to identify and understanding current best VM practices in China's construction industry for developing an implementation framework. From preceding discussion, it has been recognised that best practices cannot be identified only through the way of measuring performance with 'hard' metrics and the analysis of isolated completed $\mathrm{VM}$ case. The research team decided to identify best practices of $\mathrm{VM}$ through investigating best VM studies provided by best in class VM practitioners in the industry. Therefore, the selection of best in class VM practitioners in the industry is the first and crucial step for the benchmarking of VM. After the selection of benchmarking partners, the benchmarking of VM are conducted based on best VM case studies provided by partners. Since both "soft" and "hard" metrics were employed, self-assessment by VM practitioners, investigators' observation and document analysis were used together to get unbiased data.

\section{Analysis stage}

Data collected through interviews, questionnaires and case studies were used to make explicit VM methodology illustrated by the national standard. After that, the critical success factors for VM studies were identified and examined including techniques within each factors and the relationship among these factors. Useful observations and discussions based on the benchmarking findings were conducted to indicate how to reach best level of VM practicc.

\section{Integration stage}

Findings from the benchmarking study and experience learned from overseas VM practice guidance notes, standards, manuals and influential books ware formed together to devclop a VM implementation framework in the context of China's construction industry. Some issues closely related to the framework were discussed.

\section{Action stage}

Validation of the framework was undertaken through seminars comprising representatives from all sectors, including construction professionals, clients and VM experts. The framework went through two rounds of refinement and improvement and was finally accepted as an appreciable framework for implementing VM in China's construction industry.

\section{THE EXISTING BENCHMARKING PARTNER SELECTION APPROACHES}

The size of the areas of the pyramid in Figure 1 indicates the number of different hierarchy of partners. The upper area of the pyramid represents the partners that can be labelled best-inclass. Below that is a larger area representing what might be called best practice. The size of the areas in the pyramid indicates the probability of finding a partner in the different groups. In order to find suitable partners for benchmarking, it is necessary to apply a systematic 
approach to identify qualified partners and ensure the benchmarking result representing the level of best in class.

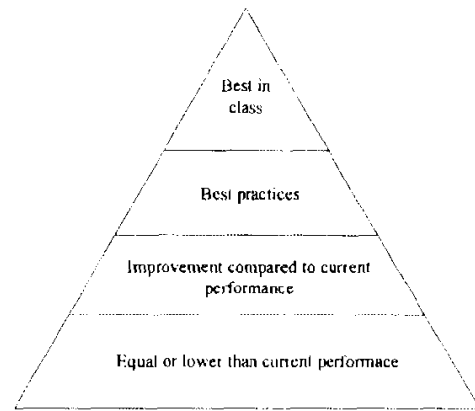

Figure 1 The pyramid of different partners (Spendolini, 1992)

Before introducing the benchmarking of VM, it is worth clarifying two terms i.e. best practices and best in class. These two terms frequently emerge in the literature of benchmarking. Usually, 'best practices' means the level of excellence for products, services, or process performance (Elmuti and Kathawala, 1997); 'best in class', whose synonym is best of breed, means outstanding performance in a field within an industry (Watson, 1993). In this paper, best practices of VM refer to the most successful VM activities while best in class VM users represent first class VM practitioners in the industry.

In order to identify best in class benchmarking partners, it is always useful to develop a long list of potential benchmarking partners for co-operative benchmarking initially. Some potential partners, who may not be interested, not have the time, or not wish to share information, will be eliminated from the initial list after the co-operative benchmarking. In order to obtain the initial list of potential benchmarking partners, there are a number of approaches to discover who is best at the process one wants to measure. Some of the sources for identifying benchmarking partners are:

- award winners;

- newspaper and magazine articles;

- journal articles;

- conference speakers;

- industry and professional associations;

- books on well-run companies;

- consultants in the industry.

When likely candidates are initially detected, some preliminary research should be conducted to narrow the list. Information may not be available on some potential partners. In these cases, they are normally dropped from the list. Sufficient public data may be available on the ones remaining so that the benchmarking team can make a final decision on which organizations should be amongst the benchmarking partners. Although the list of potential partners has been reduced by now, it may still be difficult to find the best in class organizations to approach. The benchmarking process involves many attributes ranging from technical viewpoints to cultural aspects (Zairi, 1994). Therefore, some practitioners have developed a variety of methodologies to make this decision. The following three types of techniques have been developed for use in the selection of benchmarking partners, and are based on multi-attribute decision-making tools (MacCrimmon, 1968; Canada and Sullivan, 1989). 


\section{Alternatives to alternatives scorecard}

A scorecard is a matrix in which alternatives (candidates for benchmarking) are shown in the first row and attributes (criteria) are shown in the first column. The outcomes of each alternative are described by numbers between 0.00 to 10.0 with respect to each attribute. The best alternative for each attribute is highlighted by symbol and/or colour. Table 1 shows an example of the matrix for four alternatives attributes. In this example, if the user needed to select two candidates, candidates 1 and 4 can be eliminated as they have the least number of symbols, leaving the organization to decide between candidates 2 and 3 . The main disadvantage of this technique is that it does not take the attributes' weight into account. Furthermore, by increasing the number of alternatives and attributes, the complication of the decision increases. Therefore, the use of this technique is to narrow the candidates list in the initial step rather than to obtain the final list of benchmarking candidates.

Table 1 Alternatives to alternatives scorecard

\begin{tabular}{lccccc}
\hline & Criterion A & Criterion B & Criterion C & Criterion D & Criterion E \\
\hline Candidate 1 & $(7)$ & 7 & 6 & 5 & 4 \\
Candidate 2 & 6 & $(9)$ & 7 & $(8)$ & 6 \\
Candidate 3 & 5 & 5 & 4 & $(8)$ & $(8)$ \\
Candidate 4 & 3 & 4 & 5 & $(6)$ & 5 \\
\hline
\end{tabular}

\section{Ranking the alternatives}

For this technique, candidates are compared in pairs against each different attribute or decision criterion (Table 2). For each attribute, the practitioner must decide which of the two candidates being compared is more advanced. The preferred alternative gets allocated one full score, and in case two candidates are same, half point is given to each alternative. When all alternatives (candidates) have been compared based on all individual attributes, the results are summarized by a chart. Although this technique facilitates decision making, it does not include any priority rating for the attributes, i.e. all attributes carry the same weight in this technique. Moreover, there is no systematic method to control the consistency of the comparison, and it might become subjective during certain instances.

Table 2 Ranking the alternatives

\begin{tabular}{lcc}
\hline Criterion $\mathrm{A}$ & Criterion B & Criterion $\mathbf{C}$ \\
\hline Candidate $1>$ Candidate 2 & $\ldots \ldots . .$. \\
Candidate $2>$ Candidate 3 & $\ldots \ldots .$. \\
$\ldots . . .$. & $\ldots \ldots .$. \\
\hline
\end{tabular}

\section{(3) Polar graphs}

This is a technique most often cited by many benchmarking authors. The polar graph is designed in a way that the ideal or 'best' possible outcome of alternatives is assigned to the border of the circle (see Figure 2). The rays drawn from the centre of the circle represent an evaluation rating on a scale of 0.0 to 10.0 for different attributes (each ray representing an attribute). By connecting each alternative's outcomes to each other, different polygons are created to represent the specific candidate. The candidate that provides a web covering the larger domain is considered to be the best benchmarking partner. Figure 2 is an example of this kind of graph for two candidates. The main advantages of this technique include visual presentation and ease of decision-making. Moreover, the technique facilitates the understanding of weaknesses and strengthens of each candidate. However, it is not an effective technique when the number of alternatives is more than three or four. Therefore, it is not suitable for use when many potential candidates are taken into account. In addition, it still has the same disadvantage of previous techniques whereby all attributes have the same weight. 


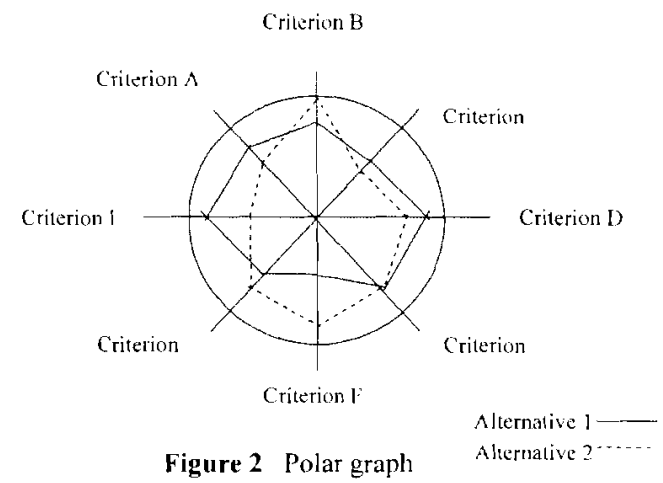

\section{THE SELECTION MODEL BASED ON AHP}

\section{The AHP approach}

Since the above techniques cannot employ the priority rate of attributes for the selection of benchmarking partners, it is necessary to develop a rigorous and systematic approach to select benchmarking candidates. The Analytic Hierarchy Process (AHP) is a procedure suited for complex technological, economic, and socio-political decision-making (Saaty, 1990). It was applied by the research team to identify ideal benchmarking partners for VM.

AHP was developed by Thomas L.Saaty in the early 1970s to help individuals and groups deal with multi-criteria decisions. By incorporating both subjective and objective data into a logical hierarchy framework, AHP provides decision-makers with intuitive or common sense approach to evaluating the importance of every element of a decision through a pair-wise comparison process (Saaty and Vargas, 1991). This method allows decision-makers to structure multi-attribute problems in the form of a hierarchy or a set of integrated levels. In general, the hierarchy has at least three levels: the goal, the criteria and the alternatives.

The process begins by determining the relative importance of the criteria in meeting the goal. Then pair-wise comparisons are made between the alternatives with respect to each criterion to decide the relative importance of one alternative versus another. The pair-wise comparison is guided by a nice-point scale as depicted in Table 3. Finally, the results of the two analyses are synthesized to compute the relative importance of the alternatives in meeting the goal. The pair-wise comparison is represented by a comparison matrix. If there are $\mathrm{n}$ items that need to be compared for a given matrix, a total of $n(n-1) / 2$ judgments are needed. The eigenvector of each pair-wise comparison matrix provides a regional priority ordering, eigenvector gives a measure of the consistency of judgment. After synthesizing the results, a global priority order of each alternative with respect to the goal is given by the synthesized cigenvector. A global consistency ratio of less than 0.10 is acceptable. Otherwise some revisions of judgments arc required.

Table 3 Pair-wise comparison scale (Adapted from Saaty 1980)

\begin{tabular}{ll}
\hline Level of importance & Definition \\
\hline 1 & Equal importance \\
3 & Weak importance of one over another \\
5 & Fssential or strong importance \\
7 & Very strong or denonstrated importance \\
9 & Absolute importance \\
$2,4,6,8$ & [ntermediate values between adjacent scale values \\
\hline
\end{tabular}




\section{The hierarchy model and Expert Choice}

According to the approach of AHP, a problem is broken down into a hierarchy with the goal at the top (Level 1). Level 2 of the hierarchy comprises the main criteria to achieve the goal, and subsequent levels consist of elements with increasing degree of detail. In this study, Expert Choice software is applied to assist in building a hierarchy model. This software is a decision support system (DSS) that enables the decision maker to resolve a multi-faceted problem in the form of a hierarchy. The design of Expert Choice is based on the AHP approach. It provides decision makers with the tools to construct decision frameworks from both routine and nonroutine problems and ways to include value judgments in these decision frameworks. Figure 3 shows the hierarchy model for the selection of VM benchmarking partners. Identifying best in class VM users is the goal and at the top level. Level 2 of the hierarchy comprises the abbreviation of main criteria to achieve the goal. Detailed explanations are provided in next section. Level 3 indicates the candidates for ideal benchmarking partners. For the sake of simplicity, candidate $i$ is abbreviated as CAD. $i$ in the blow figure.

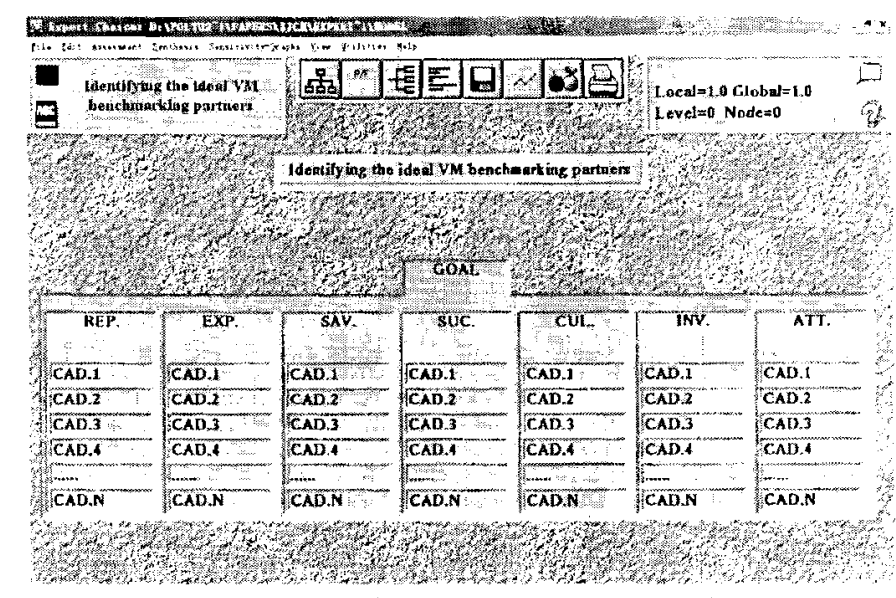

Figure 3 The hierarchy model built by Expert Choice

\section{Determination of selection criteria}

The criteria listed in level 2 of the hierarchy model are derived from comprehensive literature reviews and discussions with a number of VM experts. In cxisting literature, a number of authors mentioned a set of criteria to evaluate VM practitioners although they are not proposed in a systematic and hierarchical way. For example, Romani (1975) reported a set of key elements that led to successful value management applications, including the work experience, professional training, duties assigned to value engineers and cost of the value engineering program. Maurer (1996) argued that successful VM users should possess the characteristics of support from management sponsors, the integration of VM with the objectives of the organization, an independent VM administrator, continuously train VM facilitators, plan for VM proposal implementation, and co-operate within the organization.

Based on literature review and the knowledge of the research team, an initial list of criteria was nominated. Two rounds of discussions with 5 local VM experts were organized to finalise the evaluation criteria. In the first round of discussions, the research team asked the experts to review the initial list of criteria, delete those that are not appreciated, and suggest new criteria not included in the initial list. In the second round of discussions, the research team presented the revised list resulting from the first round of discussions to the local experts again and asked 
them to indicate which criteria should be considered in the evaluation process. According to the consensus of the research team and local VM experts, a total of 7 criteria, including both quantitative and qualitative (see Table 4), were set up to pick ideal benchmarking partners. Table 4 The criteria for selecting ideal benchmarking partners

Table 4 The criteria for selecting ideal benchmarking partners

\begin{tabular}{|c|c|c|}
\hline Criterion & bbreviation & Explanation \\
\hline 1. Reputation & Rep. & The reputation of VM application of the candidate. \\
\hline 2. VM expericncc & Exp. & $\begin{array}{l}\text { The number of VM projects the candidate implemented in } \\
\text { past } 5 \text { years. }\end{array}$ \\
\hline 3. Savings & Sav. & $\begin{array}{l}\text { The average ratio of cost savings produced by VM over the } \\
\text { cost of VM activity in past } 5 \text { years. }\end{array}$ \\
\hline 4. Success ratio & Suc. & $\begin{array}{l}\text { The ratio of clients satisfied VM cases over the total VM } \\
\text { cases conducted in recent } 5 \text { years. }\end{array}$ \\
\hline 5. VM culture & Cul. & $\begin{array}{l}\text { The history of VM application and the extent of support form } \\
\text { senior managers. }\end{array}$ \\
\hline 6. Involvement of employees & Inv. & The number of employees involved in VM studies. \\
\hline 7. Attitude & Att. & The candidate's attitude towards the benchmarking study. \\
\hline
\end{tabular}

\section{Setting up weightings with AHP approach}

The AHP approach was used in this study to determine the weighting of each selection criterion. The mathematics of AHP to calculate the relative importance weighting for the selection criteria are based on linear algebra and graph theory. The mathematical details of this approach can be found in the publications of Saaty $(1980,1988)$. In this study, the calculation of the weighting is automatically processed by Expert Choice software.

By answering the question "with respect to the goal, what is the importance of criterion i over criterion $\mathrm{j}$ ?', the research team entered the values in a comparison matrix provided by Expert Choice. The values reflect how much more important criterion $i$ is than criterion $j$. For instance, if the research team believes that 'reputation' is moderately important than 'experience', the value of 4 is entered in the grid, which is at the cross point of 'REP' row and 'EXP' column. If the research team wants to switch the relationship between 'reputation' and 'experience', pushing the 'Invert' button will express that 'experience' is moderately more important than 'reputation'. The value of 4 will also be highlighted with red font. The judgment of whole pair-wise comparison is guided by the scale system in Table 3 .

After the pair-wise comparison matrix was formed, Expert Choice then calculated the inconsistency ratio (IR). If the IR did not meet the 0.1 threshold, the research team then went back to re-examine the values assigned to each criterion and make adjustments where considered necessary. The IR was then checked again. The final matrix is shown in Figure 4 , whose IR ratio is 0.06. Based on this matrix, Expert Choice calculated weighting of each criterion automatically. The final result is illustrated in Figure 5.

\section{Ranking VM benchmarking candidates}

Having assigned weight to each of the criteria, the next step was to evaluate each candidate against these criteria. For the quantitative criteria, such as EXP, SAV and INV, the evaluation result was shown as score according to the equation:

$$
v_{i j}=\frac{a_{i j}-v_{j \text { min }}}{v_{j_{\text {max }}}-v_{j_{\text {min }}}} \quad \text { (Equation 1) }
$$




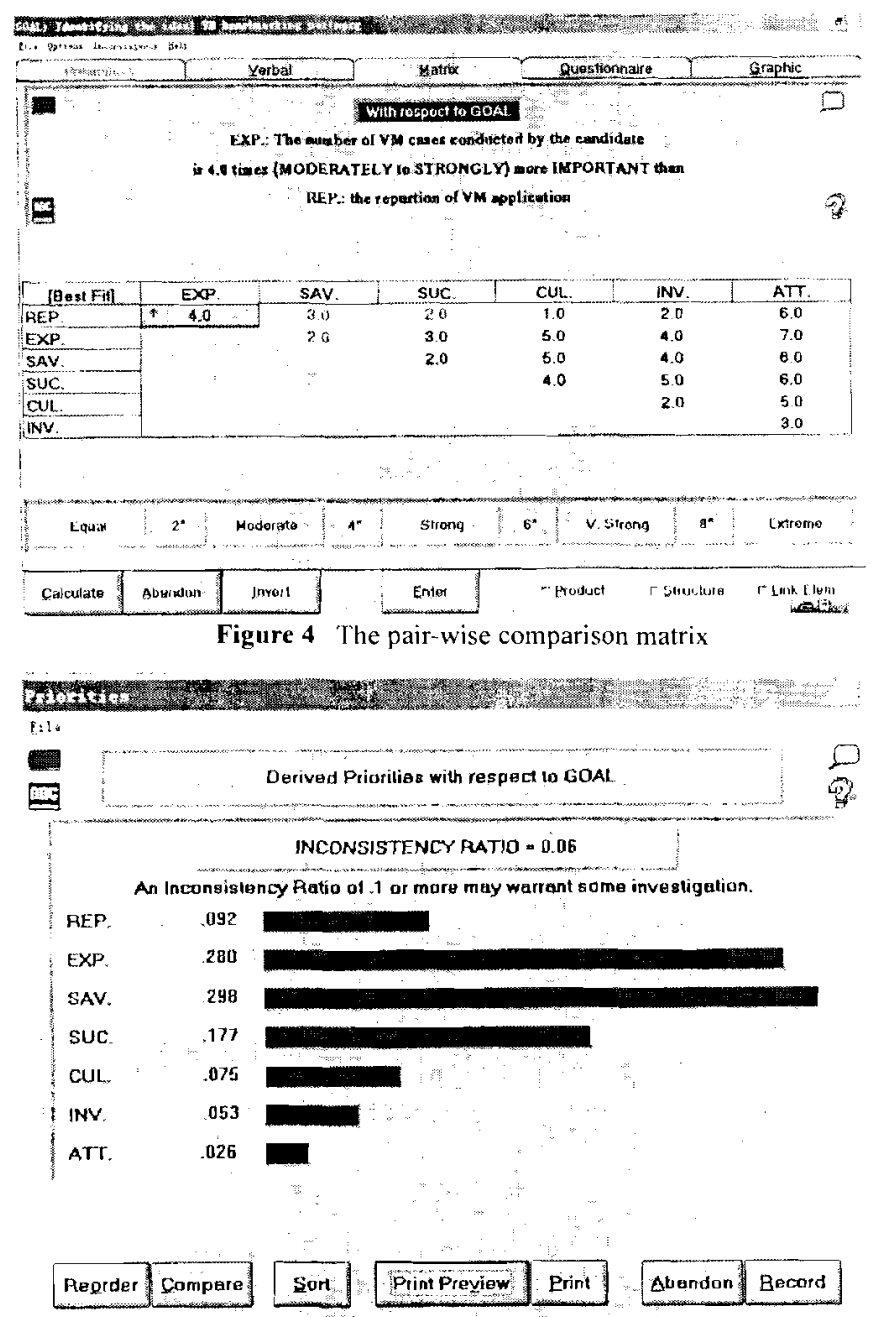

Figure 5 The weighting of each criterion

where

$\mathrm{v}_{\mathrm{ij}}$ means the value that candidate $i$ obtained against the criterion $j$;

$\mathrm{a}_{\mathrm{ij}}$ means the raw value of the candidate $i$ obtained against the criterion $j$;

$V_{j \text { max }}^{1,}$ means the maximum value obtained by any candidates against the criterion $j$;

$V_{j \text { min }}$ means the minimum value obtained by any candidates against the criterion $j$;

For example, for criterion 3 (The average ratio of cost savings produced by VM over the cost of VM activity in past 5 years), candidate $i$ is 5 . Then raw value $a_{i j}$ obtained by candidate $i$ against criterion $j$ can be directly denoted as $a_{i 3}=5$. If the maximum raw value against criterion $j$ among all candidates is 10 and the minimum raw value is 2 , then according to equation 1 , the score of $v_{i 3}=(5-2) /(10-2)=0.375$.

For qualitative REP, CUL, and ATT, the raw value of each candidate could not be decided directly. Then they were measured subjectively by the research team based on a five-point scale system: excellent- 5 , very good- 4 , good-3, fair- 2 and poor- 1 . The score was regarded as raw 
value for each candidate, and then the value $v_{i j}$ of each candidate against each of qualitative criteria also can be calculated with equation 1 .

Each score $V_{i j}$ representing the evaluation result was multiplied with the corresponding weighting of each criterion $w_{j}$. The results then were totalled as the ranking score for each candidate. The procedure can be shown as equation 2 :

$$
R_{i}=\sum_{j=1}^{7} v_{i j} \cdot w_{j}
$$

\section{Data collection and selection results}

After the establishment of model for the selection, a questionnaire was designed to collect data for measuring candidates against the criteria contained in the model. With the help of local VM associations and experts, the research team sent a copy of the questionnaire to each of 20 potential partners. Following the distribution of questionnaires, a phone call to each of potential partners was made to facilitate the return of the questionnaire and further check the partner's willingness to participate in the study. Fourteen VM users returned their completed questionnaire and were evaluated according to the model detailed in previous sections.

The evaluation results and the ranking of these fourteen candidates are listed in Table 5. Five candidates obtained the ranking score over 0.75 and were selected as ideal benchmarking partners representing best in class VM practitioners for further investigation.

\begin{tabular}{|c|c|c|c|c|c|c|c|c|c|}
\hline Candidate No. & $\begin{array}{c}\text { KEP } \\
0.092\end{array}$ & $\begin{array}{c}\text { EXP } \\
0.280\end{array}$ & $\begin{array}{c}\text { SAV } \\
0.298\end{array}$ & $\begin{array}{l}\text { SUC } \\
0.177\end{array}$ & $\begin{array}{c}\text { CUL } \\
0.075\end{array}$ & $\begin{array}{l}\text { INV } \\
0.053\end{array}$ & $\begin{array}{c}\text { ATT } \\
0.026\end{array}$ & Score & Rank \\
\hline $\mathrm{I}$. & 0.750 & 1.000 & 0.920 & 0.688 & 1.000 & 0.889 & 1.000 & 0.893 & 2 \\
\hline 2. & 0.250 & 0.417 & 0.400 & 0.000 & 0.750 & 0.667 & 0.250 & 0.357 & 10 \\
\hline 3. & 0.750 & 0.667 & 0.880 & 1.000 & 0.750 & 0.778 & 0.750 & 0.812 & 3 \\
\hline 4. & 0.500 & 0.417 & 0.200 & 0.625 & 0.250 & 0.333 & 0.500 & 0.382 & 9 \\
\hline 5. & 1.000 & 0.750 & 0.720 & 0.813 & 0.750 & 1.000 & 0.500 & 0.783 & 4 \\
\hline 6. & 0.500 & 0.250 & 0.480 & 0.000 & 0.250 & 0.444 & 0.750 & 0.321 & 11 \\
\hline 7. & 1.000 & 0.833 & 1.000 & 0.875 & 0.750 & 0.889 & 0.750 & 0.901 & 1 \\
\hline 8. & 0.000 & 0.000 & 0.400 & 0.313 & 0.500 & 0.222 & 0.500 & 0.237 & 14 \\
\hline 9. & 0.500 & 0.500 & 0.320 & 0.500 & 0.250 & 0.556 & 0.000 & 0.418 & 6 \\
\hline 10. & 0.750 & 0.750 & 0.750 & 0.813 & 0.750 & 0.778 & 0.750 & 0.763 & 5 \\
\hline 11. & 0.250 & 0.583 & 0.240 & 0.438 & 0.500 & 0.222 & 0.5000 & 0.398 & 8 \\
\hline 12. & 0.500 & 0.417 & 0.000 & 0.469 & 0.500 & 0.222 & 0.750 & 0.314 & 12 \\
\hline 1,3 & 0.250 & 0.333 & 0.220 & 0.313 & 0.250 & 0.444 & 0.500 & 0.293 & 13 \\
\hline 14. & 0.250 & 0.417 & 0.520 & 0.519 & 0.000 & 0.000 & 0.500 & 0.399 & 7 \\
\hline
\end{tabular}

\section{DISCUSSION AND CONCLUSIONS}

The selection of best in class partners is critical for the success of benchmarking. The multitude of criteria makes the selection become a difficult and complex task. An analytical model based on AHP was developed for the selection of ideal VM benchmarking partners. This model overcomes the drawbacks of previous selection methods, such as no weighting for inportance in the critcria, no systematic method to control the consistency of the comparison, and too subjective judgements during the sclection process. 
As for the assessment of importance weightings of criteria, the analytical hierarchy process improves both the objectivity and the consistency. Meanwhile, it enables the research team to take into account the characteristics of VM. The quantitative approach structured the information of candidates, thus putting the decision on a firmer footing. The use of the AHP technique requires the research team to examine all the decision criteria carefully. The checking of the inconsistency ratio also helps to minimize illogical importance weighting assignments for criteria. These all improve the objectivity of the selection of benchmarking partners.

The use of Expert Choice software accelerated the selection process and accuracy. The form of pair-wise matrix, the calculation of consistency ratio and the determination of importance weighting all can be automatically realized. The results of benchmarking study among five VM partners, who were selected with the analytical model, provided fundamental elements for the development VM implementing framework in the context of China's construction industry. The benchmarking output suggests that this analytical model is reliable. Although the analytical model was developed mainly for the selection of VM benchmarking partners, the concepts and principles could be extended easily to other benchmarking activities.

\section{ACKNOWLEDGEMENTS}

The work described in this paper was substantially supported by research grants from the Hong Kong Polytechnic University and the Research Grants Council of the Hong Kong Special Administrative Region, China (Project No: PolyU 5161/04E).

\section{REFERENCES}

Andersen, B.\& Pettcrsen, P. (1996), The Benchmarking Handbook: Step-by-step Instructions, Chapman $\&$ Hall.

APQC (1993), Organizing and Managing Benchmarking, American Productivity \& Qualin' Center; Houston, TX.

Camp, R.C. (1989), Benchmarking - the Search for Industry's Best Practice that Lead to Superior Performance, American Society of Quality Control 17, Quality Prcss, Milwaukee, WI.

Canada, J.R. and Sullivan, W.G. (1989), Economic and Multi-attribute Evaluation of Advanced Manufacturing Systems, Prentice-I lall, Englewood Cliffs, NJ.

Elmuti, D and Kathawala, Y, (1997), An overview of benchmarking process: a tool for continuous improvement and competitive advantage, Benchmorking for Quality Managenent \& Technolog;, MCB University Press, 4(4), 229-243.

Fisher, D. (1995), Benchmarking in construction industry, Journal of Management in Engineering, ASCE, 11(1), 50-57.

Garnett, N. and Pickrell, S. (2000), Benchmarking for construction: theory and practice, Construction Management and Economics, 18, 55-63.

MacCrimmon, K.R. (1986), Decision making among multiple attribute alternatives: a survey and consolidated approach, Memo RM-4823, Rand Corporation.

Male, S., Kelly, J., Fernie, S., Gronqvist, M. and Bowles, G. (1998), The Valuc Management Benchmark: Research Results of an International Benchmarking Study. London: Thomas Telford.

Maurer, J.H. (1996), Key factors in starting and maintaining a VA/VE continuous improvement program, SAVE International Conference Proceedings, SAVE, 147-152. 
McNair, C.J. and Rimmer, M. (1992), Benchmarking, A Tool For Contimuous Improvement, Harper Business Press, New York, NY.

Razmi J., Zairi M., and Jarrar Y.F. (2000), 'The application of graphical techniques in evaluating benchmarking partners, Benchmarking: An International Journal, 7 (4), 304-314

Romani, P.N. (1975), The Department of Defense Value Engineering Change Proposal Program, PhD thesis, George Washington University, USA.

Saaty, T. L. (1980), The Analytical Hierarchy Process, McGraw-Hill, New York.

Saaty, T. L. and Vargas, L. G. (1991), Prediction, Projection and Forecasting, Kluwer, Boston, MA..

Saaty, T.L. (1990), The Analytic Hierarchy Process: Planning, Priority Setting, Resource Allocation, RWS Publications, University of Pittsburgh.

Spendolini M.J. (1992), The Benchmarking Book, Amacom, New York, NY.

Watson, G.H. (1993), Strategic Benchmarking: How to Rate Your Company's Performance against the World's Best, John Wiley and Sons Inc, New York, NY.

Zairi, $M$ and Youssef, M, (1995), Benchmarking critical factors for TQM Benchmarking for Quality Management \& Technology, MCB University Press, 2(1), 5-20. 